\title{
Percepción de competencias y plan de mejora para la consolidación de los títulos de grado en educación: la mirada de los estudiantes
}

\author{
Competences perception and improvement plans for consolidating \\ degrees on education: the students' view
}

\author{
Pilar Martínez Clares, ${ }^{a}$ Natalia González Morga,${ }^{b}$ Cristina González Lorente ${ }^{c}$ \\ ${ }^{a}$ Departamento de Métodos de Investigación y Diagnóstico en Educación, Universidad de Murcia \\ Telf.: (34) 868884 061. Correo electrónico: pmclares@um.es \\ ${ }^{\mathrm{b}}$ Universidad de Murcia \\ Telf.: (34) 868884 061. Correo electrónico: natalia.gonzalez@um.es \\ ${ }^{\mathrm{c}}$ Universidad de Murcia \\ Telf.: (34) 868884 061. Correo electrónico: c.gonzalezlorente@um.es
}

\begin{abstract}
RESUMEN
Este artículo pretende valorar la formación en competencias que reciben los estudiantes de la Facultad de Educación de la Universidad de Murcia, deteniéndose en aquellas esenciales para su desempeño profesional y en sus distintos aspectos de mejora. En la investigación participan 629 estudiantes de último curso de los Grados de Educación Infantil, Primaria, Pedagogía y Educación Social, a través de un cuestionario diseñado ad hoc. Los resultados muestran la relevancia que dan los estudiantes a las competencias transversales para su práctica profesional y las diferencias significativas existentes entre su desarrollo y dominio. Además, para optimizar el sistema universitario, el alumnado identifica diferentes mejoras localizadas en la estructura del plan de estudio y el proceso enseñanza-aprendizaje.

Palabras clave: Espacio Europeo de Educación Superior, competencias transversales y específicas, Facultad de Educación, propuestas de mejora.
\end{abstract}

\section{ABSTRACT}

This article is intended to assess the students' training of competences in the Faculty of Education at the University of Murcia. Additionally, it focused on the competences, which were considered as essential for their professional performance and also the alternatives proposed to improve their degree. This study involved 629 students of the Faculty of Education. To gather information an ad hoc questionnaire was designed. Results showed the relevance of key skills for professional practice given by the students. Furthermore, the significant differences between the development and command of these competences by the participants were emphasized in the results. In order to optimize a university system the students propose a variety of improvement proposals about the structure of the curriculum and teaching-learning process are suggested.

Key Words: European Higher Education Area, transversal and specific competences, Faculty of Education, improvement proposals. 
Estudios Pedagógicos XLII, N 3: 277-292, 2016

PERCEPCIÓN DE COMPETENCIAS Y PLAN DE MEJORA PARA LA CONSOLIDACIÓN DE LOS TÍTULOS DE GRADO EN EDUCACIÓN: LA MIRADA DE LOS ESTUDIANTES

\section{INTRODUCCIÓN}

Sin una fórmula exacta para dar respuesta a las profundas transformaciones experimentadas por la sociedad actual, existe consenso al considerar la educación como un factor clave y esencial para el desarrollo, teniendo como imperativo la calidad. No hay más que observar la plena escolarización entre los más jóvenes o la diversidad en la oferta formativa para entender que buena parte de la sociedad reclame en los profesionales de la educación una formación renovada, activa, actualizada y comprometida, cuyas funciones serán decisivas en la trayectoria vital y profesional de cualquier persona.

Ante esta nueva estructura social, las universidades introducen en el espacio común europeo modificaciones sustanciales en la estructura y diseño curricular de las carreras universitarias, incluidas las titulaciones de Educación. Para Fidalgo y García (2007), entre los cambios más destacables y que mayor controversia han generado en la formación universitaria se encuentran el sistema de transferencia de créditos europeos ECTS que potencia una formación centrada en el aprendizaje del estudiante y el modelo de formación basado en competencias. Este último planteamiento, que además justifica el propósito de este trabajo, centra el objeto de la Educación Superior en una formación profesionalizadora que, según el Real Decreto 1393/2007 de Ordenación de las Enseñanza Universitarias y modificado por última vez por el RD.43/2015, se organiza en torno a competencias transversales y específicas.

Las universidades incorporan este modelo de formación dicotómico en las memorias de los títulos de Grado y delimitan el conjunto de competencias de carácter general y específico que deben desarrollarse en las aulas universitarias, fruto del esfuerzo y consenso entre instituciones nacionales, como la Agencia Nacional de Evaluación de la Calidad y Acreditación (ANECA) y las reuniones gubernamentales del proceso de Bolonia o proyectos internacionales como Tuning (González y Wagenaar, 2003). No obstante, la actualidad del debate sobre las competencias en el sistema universitario se sitúa no tanto en el proceso de diseño de los planes de estudio, sino en el interés por la funcionalidad y viabilidad de las mismas, una vez finalizadas las primeras promociones de graduados.

El Centro Europeo para el Desarrollo de la Formación Profesional (CEDEFOP, 2014) recoge la amenaza que divisan los empleadores, relativa a la distancia existente entre la enseñanza universitaria y la demanda sociolaboral; de manera similar, los potenciales profesionales de la educación manifiestan su descontento con la formación recibida, ya que al finalizar sus estudios adolecen de una falta de integración entre el saber teórico y el saber práctico (Cardona, 2011). Estas y otras declaraciones revelan la necesidad de revisar si las competencias y contenidos que integran la formación universitaria son los adecuados para desarrollar y formar a los futuros profesionales, y por tanto si son suficientes y esenciales para la inserción socio-laboral de sus egresados.

Tras una revisión de la literatura sobre los conocimientos, habilidades, actitudes y valores esenciales que deben tener los profesionales de la educación para el desempeño profesional (Armengol et al., 2011; Valdemoros y Lucas, 2014;), se determina la necesidad de incluir en su formación el desarrollo de la dimensión intelectual, personal y social. Como señala Zabalza (2012), es en la complementariedad de estas dimensiones donde se alcanza realmente una formación integral que garantiza el equilibrio emocional, el bienestar psicológico, la satisfacción y el compromiso con su profesión, aun así, actualmente sigue predominando la dimensión cognitiva en la formación. 
En la última década son numerosas y diversas las iniciativas planteadas para consolidar este modelo de formación basado en competencias en las distintas universidades y facultades, estando la mayoría de ellas orientadas a elementos individuales del proceso formativo, como la metodología de enseñanza (Zabalza, 2011), la evaluación de competencias en el aula universitaria (Blanco, 2009) o la formación del docente para afrontar esta realidad (Lukas et al., 2014; Rodríguez et al., 2011). Sin embargo, resulta poco habitual encontrar estudios que afronten la mejora y consolidación de los títulos de Grado desde un enfoque amplio, que incluya factores externos al aula que redundan en su éxito, como el que realizan recientemente González, Arquero y Hassall (2014) apoyándose de un análisis documental y de la percepción del propio docente. De hecho, el interés emergente por mejorar los títulos de Grado desde el proceso enseñanza-aprendizaje y no tanto desde la estructura del plan de estudios, implica que la selección de contenidos, organización, gestión, coordinación y continuidad de la enseñanza sean aspectos que raras veces se contemplan en las investigaciones.

Atendiendo a estas carencias, la principal contribución de este trabajo es analizar el impacto y acogida del proceso de Bolonia en la Educación Superior desde un enfoque global, contemplando la experiencia vivencial de los propios estudiantes y deteniéndose en la planificación y proyección de la formación universitaria acorde con las demandas socioprofesionales. El presente trabajo traslada estas inquietudes al contexto de los títulos de Grado de la Facultad de Educación de la Universidad de Murcia una vez completadas las primeras promociones de graduados y se plantea un doble objetivo: a) conocer y contrastar el desarrollo y dominio de las competencias que los estudiantes consideran esenciales para su desarrollo profesional de los títulos de Grado en Educación Primaria, Infantil, Pedagogía y Educación Social; y b) analizar las propuestas de mejora que realizan los estudiantes sobre la estructura y planificación de la formación recibida.

\section{MÉTODO}

Este trabajo se realiza desde el enfoque metodológico integrador o mixto, donde se combinan las estrategias propias del paradigma cuantitativo a través del análisis de contenido que categoriza las respuestas valorativas de los estudiantes en relación a las competencias esenciales de su título, con las potencialidades del paradigma cualitativo durante el análisis de las propuestas de mejora desde las premisas de la teoría fundamentada. Finalmente, este estudio recurre a un diseño de investigación no experimental, descriptivo y transversal para su desarrollo.

\subsection{PARTICIPANTES}

En esta investigación participa el alumnado de $4^{\circ}$ de Grado de la Facultad de Educación de la Universidad de Murcia. Para la selección de los participantes, se realiza un muestreo aleatorio simple con el que se obtiene un total de 629 estudiantes, distribuidos como representa la Tabla 1. 
Tabla 1. Descripción de los participantes

\begin{tabular}{|l|c|c|}
\hline \multicolumn{1}{|c|}{$\begin{array}{c}\text { Títulos de Grado de Ciencias } \\
\text { de la Educación }\end{array}$} & $\mathrm{N}^{\mathrm{o}}$ participantes & $\begin{array}{c}\text { Nivel de confianza }+ \\
\text { error muestral }\end{array}$ \\
\hline Grado en Educación Primaria & 319 & $\alpha=.01+5 \%$ \\
\hline Grado en Educación Infantil & 158 & $\alpha=.05+5 \%$ \\
\hline Grado en Pedagogía & 92 & $\alpha=.05+5 \%$ \\
\hline Grado en Educación Social & 60 & $\alpha=.1+7 \%$ \\
\hline
\end{tabular}

Esta muestra se considera representativa del último curso de los títulos de Grado de la Facultad de Educación (ver Tabla 1). Del total de participantes, la edad media se sitúa en 22.97 años; el $85.2 \%$ corresponde a mujeres.

\subsection{INSTRUMENTO}

Para la recogida de información se utiliza el método encuesta o survey y como técnica, un cuestionario diseñado ad-hoc, denominado Cuestionario de Evaluación de Competencias de Grado (CECGRA). A continuación se muestra (Tabla 2) la estructura y agrupación de ítems del cuestionario.

Tabla 2. Estructura Cuestionario de Evaluación de Competencias de Grado (CECGRA)

\begin{tabular}{|c|c|}
\hline Bloques & Contenido \\
\hline B.I. Datos personales & Edad y sexo \\
\hline B.II. Competencias & $\begin{array}{l}\text { Conjunto de competencias recogidas en el plan de estudios de los } \\
\text { títulos de Grado participantes, agrupadas en cuatro tipos: } \\
\text { - Competencias Básicas del MECES (2011) y el RD.1393/2007 } \\
\text { (CGM) } \\
\text { - Competencias Transversales de la Universidad de Murcia (CT) } \\
\text { - Competencias Generales del título (CGT) } \\
\text { - Competencias Específicas del título (CET) }\end{array}$ \\
\hline $\begin{array}{l}\text { B.III. Desarrollo } \\
\text { formativo y profesional }\end{array}$ & $\begin{array}{l}\text { Dos preguntas semi-cerradas y tres abiertas que permiten profundizar } \\
\text { en la percepción de los alumnos en relación a: } \\
\text { - Proyección profesional del estudiante de Grado } \\
\text { - Competencias esenciales para su futuro profesional } \\
\text { - Competencias no contempladas en el título y que son necesarias } \\
\text { - Equilibrio entre formación recibida y requerimientos profesionales. } \\
\text { - Propuestas de mejora para el título de Grado }\end{array}$ \\
\hline
\end{tabular}


Cada una de las competencias fue valorada por el estudiante en una escala tipo Likert con cinco opciones de respuesta $(1=\mathrm{Nada} 5=$ Mucho), en relación a tres aspectos: grado de desarrollo en las distintas asignaturas, dominio o nivel de adquisición del estudiante y relevancia de las competencias para su futuro profesional. El Bloque II varía en función de las competencias que integra cada título de Grado, obteniéndose en las diferentes escalas coeficientes de consistencia interna muy altos, con un alfa de Cronbach de .948 E. Primaria, .902 E. Infantil, .880 Pedagogía y .919 E. Social respectivamente.

\subsection{PROCEDIMIENTO}

El proceso seguido en esta investigación se resume en las siguientes fases. Una vez realizada la búsqueda bibliográfica y concretados los objetivos de la investigación, se diseña el instrumento de recogida de información denominado CECGRA. Para garantizar su validez de contenido se somete al procedimiento de juicio de expertos bajo el método de agregados individuales. Paralelamente, se organiza y planifica la recogida de información contactando a los tutores de cada grupo-clase. Son los propios miembros del equipo de investigación quienes se trasladan al aula para realizar una breve explicación del cuestionario e insistir en el carácter totalmente voluntario, anónimo y confidencial de los datos recogidos. Seguidamente, se realiza el análisis y la interpretación de los datos y se redacta el informe final de los resultados.

\subsection{ANÁLISIS DE LOS DATOS}

Para el análisis de las respuestas de los estudiantes se examina la información de carácter cuantitativo mediante el SPSS v.20 recurriendo a la estadística descriptiva y a la estadística inferencial para detectar diferencias significativas $(p \leq .05)$ entre las variables estudiadas (prueba de Wilcoxon). Los datos cualitativos son analizados a través del Atlas. ti v7 bajo el criterio de reducción de información temático y una estrategia inductiva. Además se establecen las relaciones conceptuales entre los códigos resultantes en una red de contenido o network.

\section{RESULTADOS}

Los resultados de este trabajo se estructuran en dos apartados principales para facilitar su presentación y comprensión, tal y como se detallan a continuación.

\subsection{COMPETENCIAS ESENCIALES DE LOS TÍTULOS DE GRADO DE LA FACULTAD DE EDUCACIÓN DE LA UM: SU DESARROLLO Y EL DOMINIO DEL ESTUDIANTE}

El análisis de las competencias que los estudiantes consideran esenciales de su título para su desarrollo profesional muestra una amplia diversidad y heterogeneidad de respuestas, pues la mayoría de las competencias son elegidas al menos una vez por los estudiantes. Para solventar esta situación, se cree pertinente seleccionar aquellas que obtienen un porcentaje igual o superior a $10 \%$, recogidas en las tablas 3, 4, 5 y 6 . Una vez delimitadas estas competencias esenciales, se analizan en relación a dos criterios: su 
Estudios Pedagógicos XLII, N 3: 277-292, 2016

PERCEPCIÓN DE COMPETENCIAS Y PLAN DE MEJORA PARA LA CONSOLIDACIÓN DE LOS TÍTULOS DE GRADO EN EDUCACIÓN: LA MIRADA DE LOS ESTUDIANTES

desarrollo durante la formación universitaria y el dominio que presenta el estudiante. A continuación se organizan los resultados en función del título de Grado.

\subsubsection{Grado en Educación Primaria}

En el Grado de Educación Primaria se identifican nueve competencias esenciales de las treinta y una que presenta la memoria del título, representadas en la Tabla 3. Cabe destacar el predominio de las competencias transversales, la competencia CT6 es la que obtiene un desarrollo más elevado a lo largo de la formación universitaria y la más dominada por los estudiantes según su propia percepción, con una media en ambos casos superior a 4 . Por el contrario, la CT2, relacionada con el aprendizaje de una lengua extranjera, es la competencia con el valor más bajo tanto en el desarrollo que percibe el estudiante $(\bar{X} \bar{X}$ $=2.08)$ como en su dominio $(\bar{X} \bar{X}=2.85)$.

La prueba de Wilcoxon indica diferencias significativas $(p \leq .05)$ entre todas las competencias analizadas excepto en la CT6, siendo el dominio superior al desarrollo en todas ellas, menos en la CET1 que rompe con esta tendencia con un dominio o adquisición inferior a la presencia o desarrollo de esta competencia en distintas materias del Grado.

Tabla 3. Desarrollo y dominio de las competencias esenciales del título de Educación Primaria

\begin{tabular}{|c|c|c|c|c|c|c|}
\hline \multirow{2}{*}{ Competencias esenciales Ed. Primaria } & \multirow{2}{*}{$\begin{array}{c}\text { Frecuencia } \\
\%\end{array}$} & \multicolumn{2}{|c|}{ Desarrollo } & \multicolumn{2}{|c|}{ Dominio } & \multirow{2}{*}{$\begin{array}{c}\text { Sig (p) } \\
\text { Wilcoxon }\end{array}$} \\
\hline & & M & DT & $\mathbf{M}$ & DT & \\
\hline $\begin{array}{l}\text { CET2. Diseñar, planificar y evaluar procesos de } \\
\text { enseñanza y aprendizaje individuales y en colaboración. }\end{array}$ & 23,70 & 3,51 & 1,01 & 3,62 & 0,86 & .007 \\
\hline $\begin{array}{l}\text { CT6. Trabajar en equipo y relacionarse con personas } \\
\text { del mismo o distinto ámbito profesional. }\end{array}$ & 20,13 & 4,33 & 0,87 & 4,32 & 0,74 & .745 \\
\hline $\begin{array}{l}\text { CT2. Comprender y expresarse en un idioma extranjero } \\
\text { en su ámbito disciplinar (inglés). }\end{array}$ & 17,86 & 2,08 & 0,99 & 2,85 & 1,14 & .000 \\
\hline $\begin{array}{l}\text { CT1. Expresarse correctamente en español en su ámbito } \\
\text { disciplinar y, en caso necesario, con un sistema } \\
\text { alternativo. }\end{array}$ & 15,58 & 3,64 & 1,06 & 3,92 & 0,85 & .000 \\
\hline $\begin{array}{l}\text { CET10(a). Reflexionar sobre las prácticas de aula para } \\
\text { innovar y mejorar la labor docente. }\end{array}$ & 13,96 & 2,99 & 1,21 & 3,42 & 0,95 & .000 \\
\hline $\begin{array}{l}\text { CET1. Conocer las áreas curriculares, su relación } \\
\text { interdisciplinar, los criterios de evaluación y los } \\
\text { contenidos didácticos de la Educación Primaria. }\end{array}$ & 12,66 & 4 & 0,94 & 3,79 & 0,93 & .000 \\
\hline $\begin{array}{l}\text { CET4. Diseñar y regular espacios de aprendizaje en } \\
\text { contextos de diversidad. }\end{array}$ & 12,01 & 2,86 & 1,08 & 3,1 & 1,01 & .000 \\
\hline $\begin{array}{l}\text { CET5(a). Fomentar la convivencia en el aula y fuera de } \\
\text { ella y contribuir a la resolución de problemas/conflictos. }\end{array}$ & 11,04 & 2,75 & 1,13 & 3,47 & 0,91 & .000 \\
\hline $\begin{array}{l}\text { CT5. Proyectar las competencias adquiridas para } \\
\text { promover una sociedad libre, justa, igual y plural. }\end{array}$ & 10,06 & 3,15 & 1,13 & 3,62 & 3,15 & .000 \\
\hline
\end{tabular}




\subsubsection{Grado en Educación Infantil}

Entre las competencias esenciales del Grado de Educación Infantil, la competencia que obtiene una puntuación más alta es de carácter específico (CET11) con un 37.66\% de las respuesta de los estudiantes. Además, es importante destacar las similitudes encontradas en el significado de las competencias específicas de los títulos de Educación Infantil y Primaria que hacen alusión a los contenidos curriculares, atención a la diversidad y mejora de la labor docente, así como en las competencias transversales CT2 y CT6, tal y como se aprecia en las tablas 3 y 4.

En relación a las diferencias encontradas entre el desarrollo y dominio de las competencias esenciales, se muestran significativas $(p \leq .05)$ en cinco de las once competencias (CET1, CT2, CT14, CET7 y CET13). La interpretación de estas distancias sigue la línea de los estudiantes de Educación Primaria con un dominio superior al desarrollo, exceptuando los resultados de la CET1 donde se obtiene la situación contraria. Además, esta competencia es la que adquiere la valoración más alta tanto en su desarrollo $(\bar{X} \bar{X}=4,57)$ como en su dominio $(\bar{X} \bar{X}=4.41)$. Por el contrario, la CT2 vuelve a presentar el valor más bajo en ambas dimensiones.

Tabla 4. Desarrollo y dominio de las competencias esenciales del título de Educación Infantil

\begin{tabular}{|c|c|c|c|c|c|c|}
\hline \multirow{2}{*}{ Competencias esenciales Ed. Infantil } & \multirow{2}{*}{$\underset{\%}{\text { Frecuencia }}$} & \multicolumn{2}{|c|}{ Desarrollo } & \multicolumn{2}{|c|}{ Dominio } & \multirow{2}{*}{$\begin{array}{l}\text { Sig }(p) \\
\text { Wilcoxon }\end{array}$} \\
\hline & & $\mathbf{M}$ & DT & $\mathbf{M}$ & DT & \\
\hline $\begin{array}{l}\text { CET11. Conocer los procesos de desarrollo psicológico, } \\
\text { de aprendizaje, de construcción de la personalidad y de } \\
\text { atención temprana en la infancia. }\end{array}$ & 37,66 & 3,71 & 0,93 & 3,69 & 0,81 & .680 \\
\hline $\begin{array}{l}\text { CET1. Conocer los objetivos, contenidos curriculares y } \\
\text { criterios de evaluación de la Educación Infantil. }\end{array}$ & 24,03 & 4,57 & 0,73 & 4,41 & 0,71 & .000 \\
\hline $\begin{array}{l}\text { CET2. Promover y facilitar los aprendizajes en la } \\
\text { infancia desde una perspectiva globalizadora. }\end{array}$ & 18,18 & 4,27 & 0,94 & 4,29 & 0,85 & .737 \\
\hline $\begin{array}{l}\text { CET6. Conocer la evolución del lenguaje en la infancia, } \\
\text { identificar posibles disfunciones y velar por su correcta } \\
\text { evolución. }\end{array}$ & 17,53 & 3,4 & 0,91 & 3,42 & 0,85 & .795 \\
\hline $\begin{array}{l}\text { CT2. Comprender y expresarse en un idioma extranjero } \\
\text { en su ámbito disciplinar (inglés). }\end{array}$ & 15,58 & 2,07 & 0,85 & 2,42 & 1,07 & .000 \\
\hline $\begin{array}{l}\text { CET3. Diseñar y regular procesos de aprendizaje en } \\
\text { contextos de diversidad. }\end{array}$ & 13,64 & 3,43 & 0,99 & 3,5 & 0,90 & .146 \\
\hline $\begin{array}{l}\text { CET14. Adquirir hábitos y destrezas de indagación } \\
\text { para innovar y mejorar la labor docente. }\end{array}$ & 13,64 & 2,83 & 1,09 & 3,14 & 1,02 & .000 \\
\hline $\begin{array}{l}\text { CET7. Aprender estrategias para fomentar en un clima } \\
\text { lúdico e inclusivo la comprensión, expresión e } \\
\text { interacción de los niños en diferentes lenguajes. }\end{array}$ & 12,34 & 3,05 & 1,04 & 3,27 & 0,94 & .000 \\
\hline
\end{tabular}




\begin{tabular}{|l|c|c|c|c|c|c|}
\hline $\begin{array}{l}\text { CET13. Aprender a aprender como formación para } \\
\text { la vida. }\end{array}$ & 12,34 & 3,22 & 1,11 & 3,53 & 0,96 & .000 \\
\hline $\begin{array}{l}\text { CGT2. Conocer el funcionamiento, organización y } \\
\text { planificación de centro y aula y las competencias } \\
\text { asignadas a los mismos. }\end{array}$ & 11,04 & 3,58 & 0,84 & 3,52 & 0,70 & .268 \\
\hline $\begin{array}{l}\text { CT6. Trabajar en equipo y relacionarse con personas } \\
\text { del mismo o distinto ámbito profesional. }\end{array}$ & 11,04 & 4,41 & 0,86 & 4,39 & 0,76 & .485 \\
\hline
\end{tabular}

\subsubsection{Grado en Pedagogía}

En los resultados obtenidos en el Grado en Pedagogía, el $40 \%$ de las respuestas de los estudiantes vuelven a considerar la CT6 como la competencia esencial para su futuro profesional. Además esta competencia coincide con aquella que presenta el desarrollo y dominio más alto según la percepción de los estudiantes. En el lado opuesto, se encuentra la CET9 con una media en ambas dimensiones de $\bar{X} \bar{X}=2.98$. No obstante, se encuentran diferencias significativas $(p \leq .05)$ entre el desarrollo y dominio de algunas de las competencias específicas del título que hacen referencia a las acciones de diagnóstico, asesoramiento y orientación que el pedagogo desempeña en su práctica profesional. En todas ellas, el desarrollo percibido es superior a su dominio, tal y como se aprecia en la Tabla 5.

Tabla 5. Desarrollo y dominio de las competencias esenciales del título de Pedagogía

\begin{tabular}{|l|c|c|c|c|c|c|}
\hline \multirow{2}{*}{ Competencias esenciales Pedagogía } & \multirow{2}{*}{$\begin{array}{c}\text { Frecuencia } \\
\text { \% }\end{array}$} & \multicolumn{2}{|c|}{ Desarrollo } & \multicolumn{2}{|c|}{ Dominio } & \multicolumn{2}{c|}{ Sig.(p) } \\
\cline { 4 - 8 } & Media & D.T. & Media & D.T. & Wilcoxon \\
\hline $\begin{array}{l}\text { CT6. Trabajar en equipo y relacionarse con } \\
\text { personas del mismo o distinto ámbito profesional. }\end{array}$ & 39,56 & 4,35 &, 844 & 4,23 &, 891 &, 070 \\
\hline $\begin{array}{l}\text { CET6. Conocer, planificar, desarrollar y valorar } \\
\text { procesos de orientación educativa, profesional, } \\
\text { laboral y ocupacional. }\end{array}$ & 31,87 & 3,50 &, 955 & 3,29 &, 846 &, 015 \\
\hline $\begin{array}{l}\text { CET5. Analizar necesidades educativas y } \\
\text { formativas y determinar propuestas de acción } \\
\text { dirigidas a personas o grupos. }\end{array}$ & 26,37 & 3,55 &, 976 & 3,49 &, 749 &, 471 \\
\hline $\begin{array}{l}\text { CET8. Conocer, planificar, desarrollar y valorar } \\
\text { procesos de análisis y diagnóstico organizativo. }\end{array}$ & 23,08 & 3,40 &, 902 & 3,22 &, 900 &, 026 \\
\hline $\begin{array}{l}\text { CET9. Conocer, planificar, desarrollar y valorar } \\
\text { procesos de asesoramiento pedagógico. }\end{array}$ & 18,68 & 2,98 & 1,058 & 2,98 &, 937 &, 982 \\
\hline
\end{tabular}




\begin{tabular}{|l|c|c|c|c|c|c|}
\hline $\begin{array}{l}\text { CGT1. Desarrollar habilidades de resolución de } \\
\text { problemas y toma de decisiones. }\end{array}$ & 17,58 & 3,39 &, 877 & 3,40 &, 742 &, 963 \\
\hline $\begin{array}{l}\text { CET3. Conocer, diseñar, desarrollar, coordinar y } \\
\text { valorar estrategias y programas educativos y } \\
\text { formativos. }\end{array}$ & 12,09 & 3,42 &, 975 & 3,16 &, 873 &, 002 \\
\hline $\begin{array}{l}\text { CGT4. Desarrollar apertura hacia el aprendizaje a } \\
\text { lo largo de la vida. }\end{array}$ & 12,09 & 3,86 & 1,012 & 3,87 &, 867 &, 823 \\
\hline $\begin{array}{l}\text { CT3. Gestionar la información y el conocimiento } \\
\text { en su ámbito disciplinar, incluyendo las TICs. }\end{array}$ & 12,09 & 3,90 &, 790 & 4,01 &, 876 &, 210 \\
\hline
\end{tabular}

\subsubsection{Grado en Educación social}

Al igual que en el Grado en Pedagogía, los estudiantes de Educación Social consideran la competencia CT6 $(34,55 \%)$ como aquella más relevante para su futura práctica profesional. Además, se trata de la más desarrollada y la más dominada por los estudiantes, de la misma forma que opinan los futuros pedagogos y maestros de Educación Primaria. Por el contrario, la CET4 es la competencia que obtiene el valor más bajo, tanto en su desarrollo $(\bar{X} \bar{X}=3.17)$ como en su dominio $(\bar{X} \bar{X}=3.31)$. Se destaca además que de las ocho competencias que los estudiantes consideran esenciales, tres aluden al diseño de proyectos de intervención y otras tres están relacionadas con la ética social y profesional.

En relación a las diferencias significativas encontradas $(p \leq .05)$ entre las dos dimensiones analizadas, cabe mencionar las distancias halladas en la CGT3, CT4, CET9(a) y CT5, con un dominio superior al desarrollo en todas ellas, como lo representa la Tabla 6.

Tabla 6. Desarrollo y dominio de las competencias esenciales del título de Educación Social

\begin{tabular}{|l|c|c|c|c|c|c|}
\hline \multirow{2}{*}{ Competencias esenciales Ed. Social } & \multirow{2}{*}{$\begin{array}{c}\text { Frecuencia } \\
\%\end{array}$} & \multicolumn{2}{|c|}{ Desarrollo } & \multicolumn{2}{|c|}{ Dominio } & \multicolumn{2}{c|}{ Sig(p) } \\
\cline { 5 - 7 } & & Media & D.T. & Media & D.T. & Wilcoxon \\
\hline $\begin{array}{l}\text { CT6. Trabajar en equipo y relacionarse con } \\
\text { personas del mismo o distinto ámbito } \\
\text { profesional. }\end{array}$ & 34,55 & 4,25 & 1,019 & 4,23 &, 789 &, 876 \\
\hline $\begin{array}{l}\text { CGT2. Analizar la realidad social y educativa } \\
\text { para la elaboración de proyectos de } \\
\text { intervención. }\end{array}$ & 29,09 & 4,03 &, 863 & 4,05 &, 746 &, 794 \\
\hline $\begin{array}{l}\text { CET3. Diseñar proyectos y estrategias de } \\
\text { actuación en los ámbitos de la educación } \\
\text { social. }\end{array}$ & 25,45 & 4,15 &, 820 & 4,08 &, 720 &, 509 \\
\hline $\begin{array}{l}\text { CGT3. Desarrollar proyectos de intervención } \\
\text { innovadores y creativos }\end{array}$ & 23,64 & 3,42 & 1,109 & 3,77 &, 851 &, 010 \\
\hline
\end{tabular}


Estudios Pedagógicos XLII, Nº 3: 277-292, 2016

PERCEPCIÓN DE COMPETENCIAS Y PLAN DE MEJORA PARA LA CONSOLIDACIÓN DE LOS TÍTULOS DE GRADO EN EDUCACIÓN: LA MIRADA DE LOS ESTUDIANTES

\begin{tabular}{|l|c|c|c|c|c|c|}
\hline $\begin{array}{l}\text { CT4. Considerar la ética y la integridad } \\
\text { intelectual en la práctica profesional. }\end{array}$ & 21,82 & 3,43 & 1,095 & 3,92 &, 869 &, 000 \\
\hline $\begin{array}{l}\text { CET4. Implementar proyectos de actuación } \\
\text { en diversos ámbitos y con diferentes agentes. }\end{array}$ & 18,18 & 3,17 & 1,117 & 3,31 & 1,087 &, 179 \\
\hline $\begin{array}{l}\text { CET9(a). Comprender y actuar de acuerdo } \\
\text { con el sentido social y ético de la educación } \\
\text { ocial. }\end{array}$ & 16,36 & 3,67 &, 968 & 4,07 &, 841 &, 002 \\
\hline $\begin{array}{l}\text { CT5. Proyectar las competencias adquiridas } \\
\text { para promover una sociedad libre, justa, igual } \\
\text { y plural. }\end{array}$ & 14,55 & 3,47 & 1,081 & 3,75 & 1,114 &, 015 \\
\hline
\end{tabular}

\subsection{PROPUESTAS DE MEJORA PARA LOS TÍTULOS DE GRADO DE LA FACULTAD DE EDUCACIÓN}

Tras realizar la categorización y codificación de las propuestas de mejora dadas por los estudiantes, se obtienen diferentes respuestas que convergen en dos grandes categorías: La “Estructura del plan de estudios" y "Planificación del proceso de enseñanza-aprendizaje”, recogidas de forma general y común a todos los Grados analizados en la red de contenido (Figura 1).

Figura 1. Red de contenido común para todos los títulos

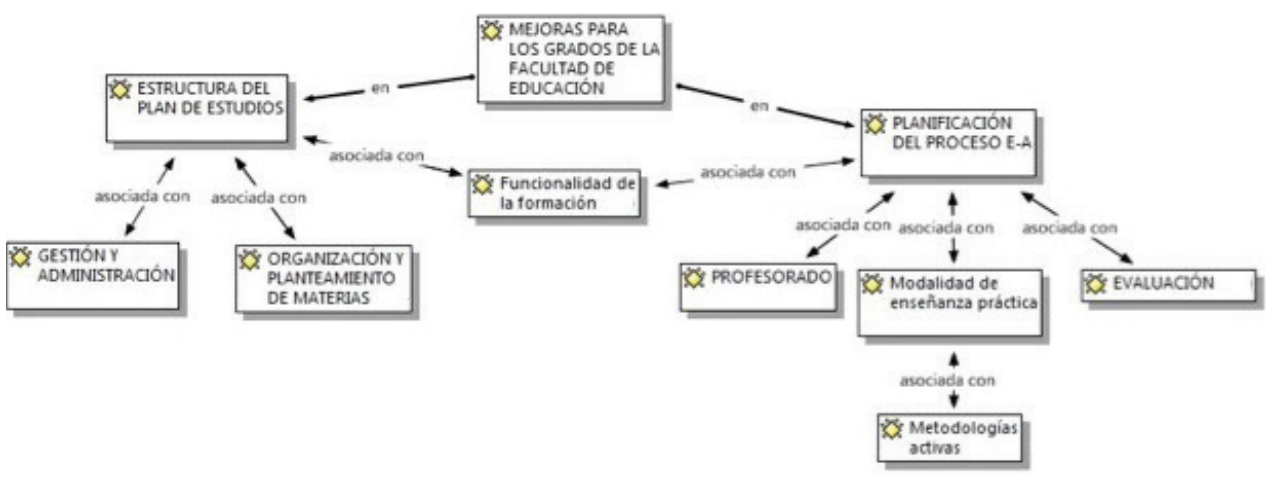

\subsubsection{Propuestas de mejoras en la estructura del plan de estudios}

La restructuración del plan de estudios es, sin duda, donde más insistencia hace el alumnado en cualquiera de las titulaciones analizadas en sus propuestas de mejora. Esta categoría incluye diferentes subcategorías que merecen especial mención. Es el caso de las propuestas planteadas en torno a la Gestión y Administración de los títulos, con "clases menos numerosas" en cuanto al número de alumnado y una mejora en la distribución de los horarios entre cuyas propuestas aparece "la disponibilidad de un día a la semana de trabajo autónomo (...) ya que apenas les queda tiempo para hacer trabajos y estudiar por su cuenta". 
La otra subcategoría denominada Organización y Planteamiento de materias recoge en todos los Grados el mayor número de iniciativas y mejoras aportadas. El alumnado señala aquí la necesidad de dedicar más horas a las prácticas externas en su formación inicial. Asimismo, también plantean la importancia de mejorar la coordinación que existe entre las diferentes asignaturas, y con ello entre los docentes encargados de impartirlas, para "evitar que los contenidos se solapen” y que "muchas de ellas hablen de lo mismo, siendo distintas entre sí"; o de reforzar el desarrollo de determinadas competencias que el alumnado considera imprescindibles para mejorar los títulos de Grado. En este sentido, cabe esperar que los estudiantes señalen diferentes competencias específicas en función de sus estudios.

En este análisis destacan las aportaciones de los estudiantes de Educación Primaria cuando afirman que "no han dado la didáctica que necesitan [...] para aprender a enseñar en un aula", en el que otros compañeros consideran que se forman como si todas fuesen "aulas perfectas", por lo que demandan aumentar las competencias relacionadas con la atención a la diversidad. Por su parte, también cabe mencionar, la percepción de algunos de los estudiantes de Educación Social cuando proponen más atención a los contextos y colectivos específicos con los que después deberán actuar como futuros profesionales de la educación y la visión de los pedagogos que demandan mejorar su formación en orientación educativa y profesional.

Además de todas estas competencias ya contempladas en los planes de estudio de cada titulación, todos los estudiantes coinciden en señalar la relevancia y necesidad de reforzar el desarrollo de competencias transversales como la creatividad, el trabajo autónomo o los idiomas. Precisamente, inciden en esta última competencia y proponen de forma reiterada, incorporar la competencia en un idioma extranjero al currículo desde el primer año de carrera.

De forma general, se citan las principales propuestas de mejora que los estudiantes realizan en torno a la estructura del plan de estudios de cada titulación, sin embargo, es importante señalar que el alumnado de Educación Primaria incluye una mejora, que hace referencia a la organización y planteamiento de las diferentes menciones que se introducen únicamente en este Grado. Estas propuestas incurren fundamentalmente en la duración de la formación más específica relacionada con la mención en la que se quieren especializar como maestros y proponen alargar esta formación en el Grado en dos años de forma que su plan de estudios quedase estructurado en "dos cursos comunes a educación primaria y dos dedicados a la mención". Además, otros estudiantes de esta titulación añaden, la posibilidad de elegir desde el segundo curso "asignaturas relacionadas con la mención que quieres hacer" para ir delimitando su itinerario formativo y su perfil profesional a través de la optatividad.

\subsubsection{Propuestas de mejoras en la planificación del proceso de enseñanza-aprendizaje}

La segunda de las dos grandes categorías diferenciadas hace referencia a la Planificación del proceso de enseñanza-aprendizaje (Figura 1). En este caso, las propuestas son comunes para todos los participantes y van dirigidas fundamentalmente a mejorar la implicación del docente y, especialmente, su competencia profesional. A este respecto, un estudiante crítico demanda al profesorado "una mayor actualización de conocimientos", así como "estar más atentos y conectados con la realidad educativa y laboral para poder aportar los conocimientos necesarios adaptados a la misma”. 
Estudios Pedagógicos XLII, N 3: 277-292, 2016

PERCEPCIÓN DE COMPETENCIAS Y PLAN DE MEJORA PARA LA CONSOLIDACIÓN DE LOS TÍTULOS DE GRADO EN EDUCACIÓN: LA MIRADA DE LOS ESTUDIANTES

Esta conexión con la realidad es, sin duda, una de las grandes metas que plantea el alumnado para conseguir mejorar su formación inicial, y en torno a ella proponen incluir en el proceso de enseñanza-aprendizaje que se desarrolla metodologías activas como parte de una modalidad de enseñanza más funcional.

Para ello, proponen "reducir el número de trabajos" que tienen que presentar para aprobar sus asignaturas y que estos "sean más creativos y orientados a su futura profesión", para que de esta forma su realización "pueda tener aplicación a la realidad escolar" y/o profesional. Consideran que las horas dedicadas a la realización de trabajos (grupales e individuales) son excesivas, lo que "provoca que se hagan rápido y sin aprender bien los conocimientos”. Como mejora proponen no únicamente reducir el número (cantidad), sino, especialmente, "dar más valor a los trabajos que se realizan" en la calificación final de cada asignatura " $y$ no tener solo en cuenta la nota final de un examen para aprobar" (ponderación). De hecho, los métodos y evidencias de evaluación también son objeto de mejora desde la percepción de estos estudiantes, aunque en menor proporción que las anteriores. Entre las mismas, destacan junto con un mayor equilibrio en las notas finales respecto a las horas dedicadas a la realización de trabajos y su repercusión en la nota final, aumentar el número de "trabajos individuales que les ayuden a la propia formación y autocrítica” y la opción de "eliminar la asistencia obligatoria".

\subsubsection{La funcionalidad de la formación como nexo entre categorías}

Una vez analizadas las mejoras que plantea el estudiante en relación a la estructura del plan de estudios y la planificación del proceso formativo, ambas categorías confluyen en un código con función de protocategoría, que hace referencia al incremento de la funcionalidad de la formación para abordar "los contenidos curriculares de un modo más práctico y enfocados al aula" y "establecer temarios adecuados a las necesidades [...] de la realidad laboral" (ver Figura 1), cuya idea se repite de forma constante entre las respuestas del alumnado de las cuatro titulaciones.

El alumnado va más allá de esta interesante demanda y plantea la complementariedad de percepciones como medida para introducir una mayor funcionalidad de la formación y un "mejor ajuste de competencias a las salidas profesionales" de cada título. A excepción de los estudiantes del Grado en Educación Primaria, gran parte del alumnado de las otras titulaciones agrega el valor de la experiencia y aportación que puedan realizar profesionales de la educación en activo, ya que "son los que realmente conocen qué aspectos son relevantes y necesarios y qué otros se pueden obviar" en los nuevos planes de estudio. Añaden, además, la importancia de "escuchar las valoraciones de los alumnos que son los que están recibiendo este sistema de enseñanza [...] y tener en cuenta otros sistemas educativos internacionales", con sus potencialidades y limitaciones. En este sentido, tanto los estudiantes de Pedagogía como de Educación Social, completan esta propuesta con la necesidad de definir el perfil profesional de ambas titulaciones que en muchos casos consideran "ambiguo" e iniciar medidas desde la universidad para "dar a conocer este perfil en las instituciones" donde pueden desempeñar su profesión y avanzar en la profesionalidad de estas titulaciones. 


\section{DISCUSIÓN Y CONCLUSIÓN}

Entre los aspectos más destacados de este estudio, cabe mencionar la importancia que los estudiantes conceden a las competencias transversales, especialmente el trabajo en equipo, que coincide con una de las mejor valoradas por los empleadores para acceder al mercado laboral (CEDEFOP, 2014; Humberg, Van der Valden y Verhagen, 2013). Esta es, a su vez, una de las competencias que el alumnado percibe como la más desarrollada a lo largo de su formación universitaria y una de las que más dominan. Datos que coinciden con otros estudios realizados en Educación Superior donde se destaca el enriquecimiento general del aprendizaje de los alumnos universitarios en esta competencia (Torrelles et al., 2011) o en su alto desarrollo y dominio como sucede en este trabajo u otros realizados en el marco nacional (Ibarra y Rodríguez, 2011).

No ocurre lo mismo cuando el alumnado hace referencia a la competencia en un idioma extranjero, ya que los universitarios indican que dicha competencia es una de las que menos desarrollan en clase y también la que menos dominan, al igual que sucede en un estudio realizado por Gómez-López, Solaz y Sanjosé (2014), donde la mayoría de los universitarios de la muestra, casi el $80 \%$, tienen un nivel A2 o B1 de inglés según los estándares europeos, con grandes carencias para comprender textos universitarios en inglés. Es importante señalar que la Facultad de Educación de la Universidad de Murcia, al igual que otras universidades de España está cuidando está competencia y ha creado en los títulos de Infantil y Primaria un grupo bilingüe con tendencia a extenderlo a los otros títulos y, en su defecto, ampliarlo.

Por otro lado, las competencias específicas también tienen cabida entre aquellas que los estudiantes de la Facultad de Educación consideran más relevantes. Sin embargo, los resultados obtenidos en el desarrollo de este tipo de competencias difiere, en la mayoría de casos, de la importancia que le conceden los estudiantes, por lo que resulta necesario potenciar la formación específica de los universitarios para que las nuevas directrices educativas introducidas en la Educación Superior con el proceso de convergencia europea tengan una aplicación real y eficaz en las aulas (Hess y Warren, 2008; Méndez, Méndez y Fernández, 2015).

Para alcanzar mejores resultados en el desarrollo y dominio de las competencias, es necesario valorar la estructura y planificación curricular de los títulos de Grado. A este respecto, el propio alumnado plantea en este trabajo diferentes alternativas para mejorar dichos aspectos en su formación, como ya lo hicieran en otros estudios similares Marqués y Castaño (2014), González et al. (2014), u otros realizados desde el punto de vista de los responsables de las comisiones de garantía de calidad de grados (Pozo y Bretones, 2015). Entre estas sugerencias y actuaciones para mejorar los títulos de la Facultad de Educación se señalan, a modo de resumen, algunas de las más destacadas:

- Coordinación entre materias. En este sentido, aparecen estudios en los que el alumnado manifiesta la necesidad de coordinación docente para evitar el solapamiento de contenidos y asignaturas (Sayós et al., 2014) y se proponen nuevas alternativas a esta situación, como establecer distintos niveles de interacción entre los mismos (McAlpine, 2008) o un trabajo por equipos docentes (Rué y Lodeiro, 2010). En cualquier caso, se trata de procurar que las acciones aisladas de profesores comprometidos con el cambio en la Educación Superior y el alumnado puedan enriquecerse de un plan de estudios coherente e interrelacionado. 
- Formación del profesorado. Los estudiantes que participan en este trabajo reclaman una mayor implicación del docente y, especialmente, de su competencia profesional. Para alcanzar dichas mejoras, en la línea que plantean Ion y Cano (2012) y González et al. (2014) sería adecuado potenciar su formación continua para mejorar el desarrollo de las competencias en el aula y reducir la percepción de estos estudiantes de falta de implicación en la docencia y actualización de su formación.

- Metodologías activas y nuevas formas de evaluación. Los estudiantes plantean un cambio en las metodologías empleadas por los docentes universitarios junto con una mayor alternancia y coherencia con los contenidos de las estrategias utilizadas para la evaluación de materias. Esta misma propuesta es la que expresan los estudiantes en el simposio realizado en la Universidad de Oviedo para debatir las novedades que introducen los nuevos planes de estudios (Marqués y Castaño, 2014) y otras investigaciones (Sayós et al., 2014), donde se valoran positivamente las metodologías activas para comprender mejor la teoría, afrontar y resolver situaciones prácticas y reales (Gil e Ibáñez, 2013), así como el papel determinante que adopta la evaluación como elemento de apoyo al estudiante para alcanzar la plena autonomía en su aprendizaje (Gibbs y Simpson, 2009).

- $\quad$ Practicum. Tras el análisis de los resultados se puede comprobar, por un lado, el gran interés e importancia que el estudiante de estas titulaciones concede a la materia de Prácticas Externas, resultado que a su vez se repite en otros estudios (Armengol et al., 2011; GonzálezGarzón y Laorden, 2012); y por otro lado, pone en relieve la necesidad de ampliar este periodo y establecer una mayor flexibilidad a la hora de elegir los centros e instituciones donde realizarlas.

- Funcionalidad de la formación y contraste de perspectivas. El alumnado plantea acercar y establecer mayores vínculos entre la formación universitaria y la realidad socio-laboral para poder ver en sus diferentes materias la utilidad y aplicación más allá de las aulas universitarias. En un estudio reciente en el que se valora la percepción de los miembros de las comisiones de calidad de los Grados (Pozo y Bretones, 2015), se incide en la especial relevancia que puede representar disponer de la perspectiva de referentes externos para mejorar los títulos universitarios, forma similar a la opinión de los estudiantes de la Facultad de Educación en este trabajo.

- Menciones en Educación Primaria. El alumnado de Grado en Educación Primaria propone ampliar a dos años su formación especializada, conocida como menciones, a partir de la implantación del plan Bolonia, pues del mismo modo que perciben Díaz y Garrido (2012) en su estudio, la generalización en la formación de maestros de Educación Primaria y su especialización en el último año de carrera despierta en este alumnado cierta inquietud. Esta propuesta debe servir para abrir una línea de reflexión en torno a la necesidad de replantear la formación del maestro que garantice tanto su capacitación para impartir alguna de las especialidades como una enseñanza de calidad y una mayor identidad profesional.

- La importancia de detenerse en analizar los títulos universitarios una vez implantado el EEES, está en la línea de lo expuesto en el reciente informe de Serrano y Soler (2015), donde se señala que las probabilidades de empleo se concentrarán en los jóvenes más cualificados (por nivel formal de estudios y de competencias efectivamente adquiridas), mientras que los niveles formativos más bajos quedarán fuera del mercado de trabajo, resaltándose sobre esta base las desigualdades laborales sucedidas durante la crisis. Por lo que es indispensable reflexionar sobre el dominio de las competencias desarrolladas en la educación superior, buscando decrecer la brecha entre realidad universitaria (formación) y realidad socio-laboral (demanda laboral). 


\section{REFERENCIAS BIBLIOGRÁFICAS}

Armengol, C., Castro, D., Jariot, M., Massot, M., \& Sala, J. (2011). El Practicum en el Espacio Europeo de Educación Superior (EEES): mapa de competencias del profesional de la educación. Revista de Educación, 354, 71-98.

Blanco, A. (Coord.). (2009). Desarrollo y evaluación de competencias en educación superior. Madrid: Narcea Ediciones.

Cardona, J. (2011). Hacia la mejora de la formación práctica del estudiante de pedagogía en la UNED. Educación XXI, 14, 303-330.

CEDEFOP. (2014). Desajuste de competencias: más de los que parece a simple vista (Nota informativa). Centro Europeo para el Desarrollo de la Formación Profesional.

Díaz, M. S., \& Garrido, M. (2012). El nuevo grado de maestro y la relevancia de las asignaturas específicas para optar a la mención de educación física en las universidades andaluzas. Revista Wanceulen EF Digital, 9, 55-83. Recuperado de http://www.wanceulen.com/ef-digital

Fidalgo, R., \& García, J. (2007). Las directrices del Espacio Europeo de Educación Superior en el marco legislativo del sistema universitario español. Aula Abierta, 35, 35-48.

Gibbs, G., \& Simpson, C. (2009). Condiciones para una evaluación continuada favorecedora del aprendizaje. Barcelona: Octaedro-ICE.

Gil, P., \& Ibáñez, A. (2013). Percepción de utilidad y grado de satisfacción del alumnado de formación del profesorado con el Método del Caso. Aula Abierta, 41(3), 79-90.

Gómez-López, A., Solaz, J. J., \& Sanjosé, V. (2014). Competencia en lengua inglesa de estudiantes universitarios españoles en el contexto del EEES: nivel de dominio lingüístico, estrategias meta cognitivas y hábitos lectores. Revista de Educación, 363, 154-183.

González-Garzón, M M L., \& La orden, C. (2012). El Prácticum en la formación inicial de los Maestros en las nuevas titulaciones de Educación Infantil y Primaria. El punto de vista de profesores y estudiantes. Pulso, 35, 131-154.

González, J. M., Arquero, J. L., \& Hassall, T. (2014). Consolidación de la formación por competencias en la universidad española: estudio de un caso. Educación XX1, 17(2), 145-168.

González, J., \& Wagenaar, R. (2003). Tuning educational structures in Europe. Final report. Phase one. Bilbao: University of Deusto.

Hess, D., \& Warren, D.E. (2008). The meaning and meaningfulness of corporate social initiatives. Business and Society Review, 113(2), 163-197.

Humberg, M., Van der Valden, R., \& Verhagen, A. (2013). The Employability of Higher Education Graduates: The Employers' Perspective. Maastricht: European Union.

Ion, G., \& Cano, E. (2012). La formación del profesorado universitario para la implementación de la evaluación por competencias. Educación XX1. Revista de la Facultad de Educación, 15(2), 249-270.

Ibarra, Ma S., \& Rodríguez, G. (2011). Aprendizaje autónomo y trabajo en equipo: reflexiones desde la competencia percibida por los estudiantes universitarios. Revista Electrónica Interuniversitaria de Formación del Profesorado (REIFOP), 14 (4). Recuperado de http://www.aufop.com/aufop/ uploaded_files/articulos/1327436370.pdf

Lukas, J. F., Santiago, K., Etxeberria, J., \& Lizasoain, L. (2014). Adaptación al espacio europeo de educación superior de un cuestionario de opinión del alumnado sobre la docencia de su profesorado. RELIEVE- Revista Electrónica de Investigación y Evaluación Educativa, 20(1), $1-20$.

Marqués, M., \& Castaño, Mª A. (2014). Propuestas de mejora a partir de las opiniones de los estudiantes. Taller XX JENUI. Actas del Simposio Oviedo (pp. 3-10).

McAlpine, L. (2008). Educational teams: making teaching less private. Conferencia impartida en el V Congreso Internacional de Docencia Universitaria (CIDUI). Lleida, España.

Méndez, D., Méndez, A., \& Fernández, F.J. (2015). Análisis y valoración del proceso de incorporación 
de las Competencias Básicas en Educación Primaria. Revista de Investigación Educativa, 33(1), 233-246.

Pozo, C., \& Bretones, B. (2015). Dificultades y retos en la implantación de los títulos de grado en las universidades españolas. Revista de Educación, 367, 147-172.

Real Decreto 43/2015, de 2 de febrero, por el que se modifica el Real Decreto 1393/2007, de 29 de octubre, por el que se establece la ordenación de las enseñanzas universitarias oficiales, y el Real Decreto 99/2011, de 28 de enero, por el que se regulan las enseñanzas oficiales de doctorado (BOE, de 3 de febrero).

Rodríguez, J., Álvarez, V., Gil, J., \& Romero, S. (2011). Necesidades del profesorado de la Universidad de Sevilla respecto a la enseñanza que requiere el EEES. Cultura \& Educación, 23, 323-340.

Rué, J., \& Lodeiro, L. (Eds.). (2010). Equipos docentes y nuevas identidades académicas. Madrid: Narcea.

Sayós, R., Pagés, T., Amador, J. A., \& Jorba, H. (2014). Ser buen docente ¿qué opinan los estudiantes de la Universidad de Barcelona? Revista Iberoamericana de Psicología y Salud, 5(2), 135-149.

Serrano, L., \& A. Soler (2015). La formación y el empleo de los jóvenes españoles. Trayectoria reciente y escenarios futuros. Fundación BBVA. Recuperado de http://www.ivie.es/downloads/2015/03/ PP_FBBVA_Ivie_jovenes_y_mercado_laboral_04_03_2015

Torrelles, C., Coiduras, J., Isus, S., Carrera, F. X., París, G., \& Cela, J. Mª (2011). Competencia de trabajo en equipo: definición y categorización. Profesorado. Revista de currículum y formación del profesorado, 5(3), 229-344.

Valdemoros, M. Á., \& Lucas, B. (2014). Competencias que configuran el perfil del docente de primaria. Análisis de la opinión del alumnado de Grado en Educación Primaria. Aula Abierta, 42(1), 53-60.

Zabalza, M. A. (2011). Metodología docente. Revista de Docencia Universitaria. REDU, 9(3), 75-98.

Zabalza, M. A. (2012). Articulación y rediseño curricular: el eterno desafío institucional. Revista de Docencia Universitaria. REDU, 10(3), 17-48. 